\title{
CHAPTER 9
}

\section{MULTI-CRITERIA DECISION MAKING TO EVALUATE QUARANTINE DISEASE CONTROL STRATEGIES}

\author{
MONIQUE C.M. MOURITS AND \\ ALFONS G.J.M. OUDE LANSINK \\ Business Economics, Social Sciences Group, Wageningen University, \\ Hollandseweg 1, $6706 \mathrm{KN}$ Wageningen, The Netherlands. \\ E-mail:Monique.Mourits@wur.nl
}

\begin{abstract}
Decision making in controlling quarantine diseases is a complex, conflicting process, characterized by a mixture of epidemiological, economic and social-ethical value judgments. Policy makers have to integrate these aspects in a consistent and transparent manner in their decision making. Multi-Criteria Decision Making (MCDM) is a tool that is capable of supporting this integration. This paper gives a general overview of available MCDM techniques and provides an application to illustrate the potential support of MCDM in choosing the control strategy that best meets all of these conflicting judgments.

In the application, various strategies to control animal quarantine diseases (such as Foot and Mouth Disease (FMD), Classical Swine Fever (CSF) and Avian Influenza (AI)) were ordered according to the preferences of various stakeholders. Considering the similarity in the complexity of controlling quarantine diseases this 'animal' application provides a good illustration of the potential use of the MCDM evaluation technique within plant disease control.

Keywords: preferences; stakeholders; epidemiology; economics; social ethics
\end{abstract}

\section{INTRODUCTION}

Decision making in controlling harmful plant diseases is a complex process involving a large range of stakeholders with different and often conflicting interests. Their views may represent the interests of the farming community, other sectors of the economy, the consumer or the environment. This may create a situation of conflicting interests, as economic motives may prevail in the views of some, while landscape, environmental or human-welfare motives may be prominent in the view of others.

Multi-Criteria Decision Making (MCDM) could support policy makers in choosing the control strategy that best meets all of these conflicting interests. MCDM techniques deal with complex problems that are characterized by any

131

A.G.J.M. Oude Lansink (ed.), New Approaches to the Economics of Plant Health, 131-144 (C) 2006 Springer. Printed in the Netherlands 
mixture of quantitative and qualitative objectives. It establishes preferences between alternatives to an explicit set of objectives and measurable criteria.

Although it is one of the most frequently applied tools within operations research and management science (Dodgson et al. 2000; Voogd 1982), MCDM methods are hardly applied in the management of quarantine disease control even though it generally improves the quality and transparency of the decision-making process. A first application in the field of animal disease control was applied in 2004 (Huirne et al. 2005). In this study various strategies to control animal quarantine diseases (such as Foot and Mouth Disease (FMD), Classical Swine Fever (CSF) and Avian Influenza (AI)) were ordered according to the preferences of various stakeholders.

With respect to the complexity of controlling diseases there is a great similarity between the plant production system and the animal production system. This paper therefore provides a description of the performed 'animal disease' MCDM analysis to illustrate the potential use of the MCDM evaluation technique within plant disease control.

The remainder of this chapter is organized as follows. Section 2 gives an overview of existing main categories of MCDM approaches. This is followed by an application of one MCDM technique to the problem of controlling contagious animal diseases such as FMD and CSF. The chapter concludes with a discussion.

\section{OVERVIEW OF MAIN CATEGORIES OF MCDM TECHNIQUES}

Multi-Criteria Decision Making is by now a well established paradigm in decision sciences. The key characteristic of this paradigm is that a decision maker does not optimize a single defined objective but aims for the achievement of satisfying levels in the goals or seeks an optimal compromise between several, often conflicting objectives (Romero and Rehman 2003). The general purpose of MCDM is to serve as an aid to thinking and decision making, but not to take the decision. MCDM techniques are capable of dealing with complex problems that are characterized by any mixture of quantitative and qualitative objectives. This is done by breaking the problems into more manageable pieces to allow data and judgments to be brought on the pieces. Next, the techniques reassemble the pieces to present a coherent overall picture to decision makers (Voogd 1982).

Several MCDM techniques have been evolved in the literature since the first seminal paper by Charnes et al. (1955). Goal Programming is the oldest and most widely applied technique. The general set up of a Goal Progamming problem is:

$$
\begin{array}{ll}
\text { Min } & \sum_{i=1}^{n}\left(n_{i}+p_{i}\right) \\
\text { s.t. } & f_{i}(x)+n_{i}-p_{i}=b_{i} \\
& x \in F
\end{array}
$$

where

$n_{i} \quad=$ negative deviational variable attached to $i$-th attribute 


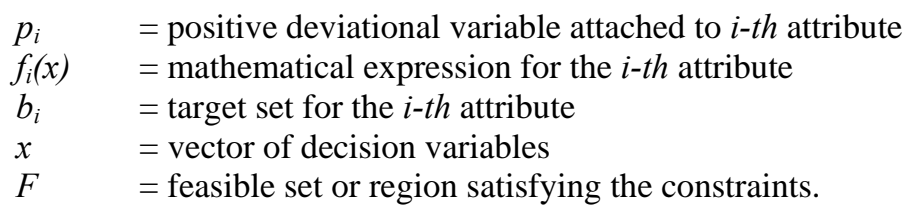

The central idea behind Goal Programming is that instead of optimizing a set of objectives (or attributes), the decision maker sets targets (b) for their achievement. Next, a solution is found by minimizing the deviations (i.e. $p_{i}$ and $n_{i}$ ) from the set of targets. The vector of decision variables is restricted by the feasible set or region $(F)$ that still satisfies the constraints.

A second main category of MCDM approaches is Multi-Objective Programming (MOP). The general set up of an MOP problem is:

$$
\begin{aligned}
& \text { Eff } Z(x)=\left[Z_{1}(x), Z_{2}(x) . ., Z_{k}(x)\right] \\
& \text { s.t. } x \in F
\end{aligned}
$$

where Eff means the search for efficient solutions in a maximizing or a minimizing sense and where $k$ objectives are involved in the search. Each of the objectives is ruled by a function $Z_{i}$. The efficient set is generated using any of three methods, i.e. a weighting method, a constraint method and a multi-criteria simplex method (Rehman and Romero 1993). The essential idea of MOP is the simultaneous optimization of several objectives and that the approach yields Pareto-efficient solutions. A solution is Pareto-efficient if another solution cannot improve it without degrading the performance of at least one other objective in the efficient solution.

Finally, a third category of MCDM approaches is based on Multi-Attribute Utility Theory (MAUT). MAUT approaches try to determine a real-value function, i.e. a utility function for a finite set of alternative systems $x^{I}, x^{2}, \ldots, x^{m}$ such that

$$
\begin{aligned}
& U\left[Z_{1}\left(x^{j}\right), Z_{2}\left(x^{j}\right) . ., Z_{k}\left(x^{j}\right)\right] \succ U\left[Z_{1}\left(x^{i}\right), . Z_{2}\left(x^{i}, . ., Z_{k}\left(x^{i}\right)\right]\right. \\
& x^{j} \succ x^{i}
\end{aligned}
$$

where $\succ$ indicates preference of system $x^{j}$ with respect to system $x^{i}$. MAUT is based on the assumptions of perfect rationality underlying the classic von Neumann and Morgenstern utility paradigm. A key assumption in the MAUT approaches is the assumption of preferential independence of objectives, meaning that the trade-off between objectives $Z_{i}(x)$ and $Z_{j}(x)$ is not affected by the level of $Z_{k}(x)$ and $k \neq i$, $j$. In many situations, this preferential independence is too strong; this is particularly obvious in the case where interactions between objectives are apparent. MAUT approaches are generally used in situations where the number of alternatives is small and where the assumption of preferential independence is not problematic.

The simplest operational form of MAUT is based on the assumption that all attribute utility functions are linear, so that the total utility function $U$ is a simple weighted sum of the attribute measures. This assumption implies linear indifferences 
curves, which is unlikely to be realistic for a wide range of attribute measures, but can be a reasonable approximation over a relatively narrow range of measures.

\section{APPLICATION TO CONTAGIOUS ANIMAL DISEASE CONTROL}

\section{Background described MCA research}

This section presents an application of a MAUT approach based on the assumption of linear indifferences curves to the problem of controlling contagious animal diseases such as Foot and Mouth Disease and Classical Swine Fever. The MAUT application (hereafter referred to as Multi-Criteria Analysis (MCA)) was part of a large EU research project in which the consequences of outbreaks of contagious animal quarantine diseases were evaluated for various EU member states. Within this EU project, member-state-specific demographic, livestock production, epidemiological and economic data were collected. These data were used as inputs in various modelling modules to obtain insight into the epidemiological and economic impact of outbreaks of contagious animal diseases. The results of these modelling studies along with the results of a detailed questionnaire to elicit the preferences of various stakeholders served as inputs of the MCA framework (Huirne et al. 2005).

\section{Steps within MCA}

The applied MCA involves eight steps, as represented by Table 1 and described below.

Table 1. The 8 steps within the applied Multi-Criteria Analysis.

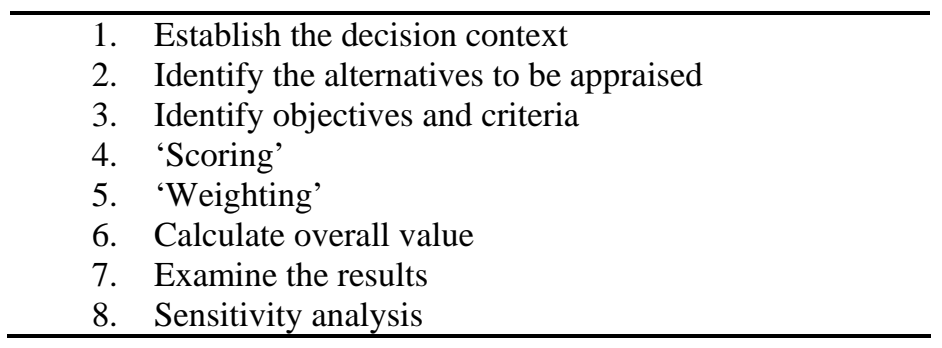

Step 1. Establish the decision context

Within this first step the objective of the MCA should be clearly defined along with an identification of the key players or so-called stakeholders; i.e., decision makers as well as people who may be affected by the decision.

MCA is all about multiple conflicting objectives. There are ultimately trade-offs to be made. Nonetheless, in applying MCA it is important to identify a single highlevel objective for which there will be sub-objectives. The aim of this MCA is to make best use of data currently available to support the decision on controlling 
contagious animal diseases like FMD, CSF and AI.

A key player or stakeholder is anyone who can make a useful and significant contribution to the MCA. Stakeholders are chosen to represent all important perspectives on the subject of the analysis. One important perspective in the field of controlling contagious animal diseases is that of the final decision maker and the animal-health authority to whom the person is accountable. Within this analysis the European Chief Veterinary Officers (CVOs) were asked to express these governmental values through questionnaires. The responses were obtained from a written questionnaire, so there was no interaction or exchange of information/experiences between the various participating CVOs. Beside the group of CVOs, two other groups of stakeholders were asked for their judgments, i.e. an agricultural interest group and a non-agricultural interest group.

Step 2. Identify the alternatives to be appraised

The appraised alternatives per contagious animal disease consisted of the default EU measures (viz. stamping out of detected herds and installation of protection and surveillance zones) and one or more of the following additional control measures:

PRE

$=$ pre-emptive slaughter of neighbouring farms within a predefined radius around a detected farm. This measure results in a regaining of the disease-free status (or removal of export bans) 3 months after culling the last detected animal.

VAC_kill $\quad=$ suppressive vaccination within a predefined radius around a detected farm. Vaccination is applied as a suppressive measure; all vaccinated animals will therefore be slaughtered as soon as the epidemic is under control. This measure results in a regaining of the disease-free status 3 months after culling the last detected or vaccinated animal.

VAC_live $\quad=$ protective vaccination within a predefined radius around a detected farm. Vaccination is applied as a protective measure; all vaccinated animals will therefore stay on the farm as soon as the epidemic is under control. This measure results in a regaining of the disease-free status 6 months after culling the last detected animal.

Step 3. Identify objectives and criteria

Assessing alternatives requires thought about the consequences of the alternatives, for strictly speaking it is the consequences that are being assessed, not the alternatives themselves. Criteria and sub-criteria or indicators are the measures of performance by which the alternative control strategies are judged. Criteria are specific, measurable objectives. They are children of higher-level parent objectives, who themselves may be the children of even higher-level parent objectives.

This research is centred on 3 high-level objectives or main criteria, viz. epidemiology, economics and social ethics. Each criterion is broken down into lower-level objectives or indicators to facilitate the scoring process. These clusters of indicators are as presented in Table 2. 
Table 2. Overview of main criteria and their indicators, along with the preference weights indicated by the CVOS

\begin{tabular}{|c|c|c|c|}
\hline Main criteria & $\begin{array}{l}\text { CVO } \\
\text { weight }\end{array}$ & Cluster of epidemiological indicators & $\begin{array}{l}\text { CVO } \\
\text { weight }\end{array}$ \\
\hline Epidemiology & 53 & Duration & 28 \\
\hline Economics & 30 & Number of infected herds & 25 \\
\hline Social ethics & 17 & $\begin{array}{l}\text { Size of affected region } \\
\text { Number of destroyed animals }\end{array}$ & $\begin{array}{l}19 \\
12\end{array}$ \\
\hline Cluster of social-ethical & CVO & Number of destroyed herds & \\
\hline indicators & weight & Number of destroyed non-farm animals & $\begin{array}{l}12 \\
5\end{array}$ \\
\hline Efficacy & 18 & & \\
\hline Socio-economic factors & 11 & Cluster of economic indicators & $\begin{array}{l}\mathrm{CVO} \\
\text { weight }\end{array}$ \\
\hline Macro-economic factors & 7 & & \\
\hline $\begin{array}{l}\text { Commercially interested } \\
\text { parties }\end{array}$ & 8 & Direct farm losses & 15 \\
\hline Animal health & 8 & Cons. farm losses in affected region & 14 \\
\hline Animal welfare & 7 & Cons. farm losses outside affected region & 10 \\
\hline Tourism & 4 & Losses of other participants & 11 \\
\hline Non-farm animals & 3 & Losses of non-agricultural sectors & 9 \\
\hline Human health & 11 & Organisation costs & 11 \\
\hline Governmental policy & 8 & Export restrictions for EU markets & 12 \\
\hline Natural life cycle & 6 & Export restrictions for non-EU markets & 9 \\
\hline Food source & 9 & Tax payer & 9 \\
\hline
\end{tabular}

In general, criteria and indicators are defined by help of the stakeholders in an iterative way. However, within the scope of this research, it was not possible to conduct such an extensive, iterative process. The definitions of criteria and indicators are therefore based 1) on the results of a former study in which Dutch stakeholders were interviewed by means of a Group Decision Room session to define the criteria by which animal control strategies should be evaluated (Huirne 2002), and 2) on additional expert consultation.

\section{Step 4. 'Scoring'}

When determining criterion scores, specific attention should be paid to the measurement scale. A distinction can be made between a quantitative and a qualitative measurement scale. In case of a quantitative scale, the measurement unit is known, i.e. a quantity has been defined as a standard by which the magnitude of differences can be expressed. Examples of quantitative measurement units are animals, farms, days, and so forth.

The measurement unit of a qualitative measurement scale is unknown. Three qualitative measurement scales can be distinguished with the ordinal scale; having the highest information content, as the numbers of this scale give a rank order. An ordinal scale expresses whether a certain choice possibility is worse or better than any other choice possibility; however, it does not say by how much. 
Even if the criterion scores have been determined on a quantitative measurement scale for all criteria, these scores are mutually incomparable since most of the measurement units differ from each other. One criterion might be expressed in number of farms, whereas another criterion is measured in days. To make the various criterion scores comparable, it is necessary to transform them into one common measurement unit by taking care that for each criterion the scores will get a range from 0 to 1 . This kind of transformation is called standardization. The method of standardization used for the scores in this study can be written as:

$$
\text { Standardized score } i=\text { (score } i / \text { maximum score) }
$$

or each score is divided by the highest score of the criterion concerned. An example is given in Table 3.

Table 3. A numerical example of the method of standardization

\begin{tabular}{lllll}
\hline Criterion 'expected length of epidemic' & \multicolumn{2}{l}{ Alternative } & & \\
\cline { 2 - 5 } & $\mathrm{A}$ & $\mathrm{B}$ & $\mathrm{C}$ & $\mathrm{D}$ \\
\hline Score (days) & 76 & 235 & 178 & 156 \\
Standardized score & 0.32 & 1.00 & 0.76 & 0.66 \\
Directed standardized score & 0.68 & 0.00 & 0.24 & 0.34 \\
\hline
\end{tabular}

An issue related to standardization is the issue of the direction of the criterion scores. For some criteria, a higher score implies an improvement, whereas for other criteria a higher score implies a deterioration. The example criterion 'length of epidemic' from Table 3 is an example of the latter. Each standardization should therefore be accompanied by a consideration of the direction of the scores. In this study, the worst criterion score is given a standardized value of 0 , whereas the best criterion score has a standardized value of 1 .

Criterion scores can be derived in many different ways. In this study all quantitative scores are based on the results of stochastic simulation modelling studies (Huirne et al. 2005). The presented MCA analyses are directed towards the 95-percentile model results, assuming a risk-averse attitude with respect to the contagious animal disease control. The scores of qualitative indicators are obtained by ranking the alternatives per criterion according to their expected effectiveness. These effectiveness rankings are based on the insights obtained from questionnaires, personal interviews and model studies.

Step 5. 'Weighting'

An indicator's weight (as well as a criterion's weight) should depend on the range of difference in the indicator scores and on how much the stakeholders care about the difference. For instance, most stakeholders consider the length of the epidemic an important decision indicator. However, when alternative strategies would result in an expected duration difference of only a few days, length would not longer be an important decision indicator. In this study, stakeholders were asked to express their 
judgments (= weights) on grounds of their subjective knowledge on possible ranges of indicator scores.

The weighting factors applied in this study are based on the results of a written questionnaire. By this questionnaire various groups of stakeholders expressed their judgments using comparative rating scales. Stakeholders had to make judgments of each indicator with direct reference to their judgments of the remaining indicators (Churchill 1995), by dividing 100 points per cluster. In this paper, the main emphasis is on the judgments of the CVOs.

\section{Step 6. Combine the weights and scores for each alternative to derive an overall} value

There are several methods by which an alternative's performance across indicators can be aggregated to form an overall assessment. Two of the most applied methods are the simple linear additive evaluation method and the concordance analysis method. The simple linear additive evaluation method combines the alternative's values into one overall value by multiplying the value score on each criterion by the weight of that criterion, followed by a summation of all those weighted scores (Dodgson et al. 2000; Voogd 1982). This method is perhaps the simplest and most intuitive of all aggregation methods. However, the method is only suitable to aggregate scores within a corresponding measurement scale (quantitative or qualitative). The concordance analysis is an evaluation method in which the alternatives are ranked by means of their pairwise comparisons in relation to the defined criteria (Nijkamp et al. 1990). Due to the pairwise comparisons, this method is able to aggregate quantitative as well as qualitative scores into one overall evaluation value.

By means of the simple linear additive evaluation method, the overall weighted scores of the three main criteria, epidemiology, economics and social ethics, are obtained. In general, the higher the overall value, the better the alternative control strategy scored within the concerned criterion.

However, the performed multi-criteria evaluation is based on criteria that are partially assessed on a quantitative scale as well as partially on a qualitative scale. To account for the specific characteristics of both measurement scales, a mixed data multi-criteria technique is applied to determine an overall score per alternative. In this mixed data evaluation technique, which is a generalized form of the concordance analysis technique, differences in alternatives are expressed in a condensed way by means of paired comparisons. Standardized scores of each indicator are compared in pairs of the evaluated alternatives, resulting in so-called dominance scores. A positive score implies dominance of one strategy in relation to another while a negative value implies submission. A dominance measure of 0 implies an indifference between the compared strategies. By weighting these dominance scores per criteria, overall dominance scores of the three main criteria are obtained.

To compare the outcomes of the quantitative and qualitative dominance scores, the scores of the individual main criteria are standardized into the same unit. In this way the dominance scores of the quantitative criteria, epidemiology and economics, 
are comparable to the dominance score of the qualitative criterion, social ethics. By weighting these standardized dominance measures with the aggregated weights of the constituent criteria the overall dominance score per alternative is calculated, which represents the degree in which an alternative was better (or worse) than another alternative.

\section{Step 7. Examine the results}

The aggregation of the dominance scores of the three main criteria (viz., epidemiology, economics and social ethics) into one overall dominance score per alternative gives an indication of how much an alternative is appreciated over another. These overall dominance scores also determine the overall ordering of the evaluated control strategies.

Step 8. Sensitivity analysis

Sensitivity analysis provides a means of examining the extent to which the relative importance weight of each criterion/indicator makes any difference in the final results. Interest groups often differ in their views of the relative importance of the criteria (or weights) and of some scores, though weights are often the subject of more disagreement than scores. In this study special attention is given to the comparison between the ranking of alternatives based on the preferences expressed by the CVOs and the ranking based on the preferences expressed by the representatives of the general public.

Using the MCA model to examine how ranking of options might change under different weighting systems can show that, for instance, two options always come out best, though their order may shift. If the differences between these best options under different weighting systems are rather small, accepting a second best option can be shown to be associated with little loss of overall benefit.

\section{Results}

Weighting factors reflecting preferences of the CVOs

The response rate of the 25 CVOs on the written questionnaire is about $80 \%$ (i.e. 20 questionnaires). The averaged CVO weights for the three main criteria and their clusters of indicators are represented in Table 2.

With respect to the main criteria, the CVOs prefer the epidemiological criterion with an average relative weight of $53 \%$. Corresponding average weights for the economic and social-ethical main criteria are $30 \%$ and $17 \%$, respectively. Duration of the epidemic (28 \%) and the number of infected herds (25\%) are regarded as the two most important epidemiological indicators. Differences between the relative weights of economic indicators are not as profound as the epidemiological indicators. Direct farm losses (15\%) and consequential farm losses in affected region (14 \%) are regarded as the two most important economic indicators. Efficacy (18\%) and social-economic factors (12\%) are considered the most important socialethical indicators (Table 2). 
MCA application to evaluate three FMD control alternatives

This section illustrates the overall MCA results based on the evaluation of FMD control alternatives for one of the studied EU member states, characterized as a net importing, densely populated livestock area.

Overall scores of main criteria

By means of the simple linear additive evaluation method, the overall weighted scores of the three main criteria, epidemiology, economics and social ethics are obtained as demonstrated by Table 4. Based on the overall epidemiological score, the Pre strategy is preferred best, followed by the Vac_live strategy. The overall 0 score on the Vac_kill strategy indicates that - compared to the other 2 alternatives Vac_kill scores worst on all epidemiological indicators. However, the efficiency with which this strategy controls an FMD epidemic is almost equal to the efficiency of the Vac_live strategy. Due to the fact that the vaccinated animals will be killed afterwards, Vac_kill scores worst on all indicators involving number of destroyed herds or animals. These indicators, therefore, do not strictly reflect epidemiological efficiency; they also reflect a social-ethical element.

Table 4. Overall weighed scores of three evaluated FMD control alternatives per main criterion. Bold printed values reflect alternatives with highest scores (= highest rank)

\begin{tabular}{lccc}
\hline \multirow{2}{*}{ Criterion } & \multicolumn{3}{c}{ Control alternative } \\
\cline { 2 - 4 } & Pre & Vac_live & Vac_kill \\
\hline Epidemiology & $\mathbf{3 6}$ & 27 & 0 \\
Economics & 58 & 53 & $\mathbf{6 3}$ \\
Social ethics & 21 & $\mathbf{5 5}$ & 33 \\
\hline
\end{tabular}

The ranking of the alternatives based on the economic criterion demonstrates that the Vac_kill strategy is preferred above the others. However, differences in overall economic values among the alternatives are rather small, as reflected by the small difference in overall value between the first and second ranked alternatives (viz. 5 points).

The economic ranking based on the MCA may differ from the economic ranking based on the result of adding all the losses to one overall value. By utilizing subjective weighting factors, the MCA ranking is not only accounting for the size of the losses but also for, for instance, value judgments on topics as 'who is bearing the losses'.

From a social-ethical point of view, the overall score for Vac_live exceeds the other 2 alternatives. With a difference of at least 22 points, Vac_kill is evaluated as the second best option.

Overall strategy value

Table 5 demonstrates the dominance scores of the three main criteria as a result of paired comparisons of the 3 FMD control alternatives. For instance, the fourth 
column of the table describes the results of the comparison between the Vac_live strategy and the Vac_kill strategy. As reflected by the positive scores, the Vac_live strategy dominates the Vacc_kill strategy on 2 of the 3 main criteria (viz. +5.19 on Epidemiology, +0.73 on Social ethics). However, regarding the Economics criterion, the Vac_live strategy is dominated by the Vac_kill strategy (economic dominance score $=-0.57$ ).

Table 5. Criteria of dominance scores of the paired comparisons of the evaluated FMD control alternatives (e.g. EU/Pre = EU strategy compared to the Preventive culling strategy)

\begin{tabular}{lcccccc}
\hline \multicolumn{7}{c}{ Pre/V_live Pre/V_kil1 } \\
Criterion & & & & & & \\
Epidemiologe/Pre V_live/V_kill V_kill/Pre V_kill/V_live & \\
Economics & 1.75 & 6.95 & -1.75 & 5.19 & -6.95 & -5.19 \\
Social ethics & 0.28 & -0.29 & -0.28 & -0.57 & 0.29 & 0.57 \\
& -1.12 & -0.39 & 1.12 & 0.73 & 0.39 & -0.73 \\
Total & 0.92 & 6.26 & -0.92 & 5.35 & -6.26 & -5.35 \\
\hline
\end{tabular}

According to the total dominance scores the Pre strategy is favoured over the other 2 strategies; i.e. all total paired dominance scores are positive. The dominance difference with respect to the Vac_live strategy is, however, small (0.92). Vac_kill is completely dominated by the other strategies as reflected by its negative total dominance scores.

\section{CONCLUSION AND DISCUSSION}

\section{The MCA study on animal disease control}

Within the EU project various MCAs were conducted to evaluate the ranking of alternative strategies to control contagious animal diseases like FMD, CSF and AI. All analyses were based on the judgment values of the CVOs. Results showed a general tendency towards the ranking of alternatives, which in most of the cases appeared to be independent of the evaluated disease (see for detailed information Huirne et al. 2005). The general tendency can be described as follows:

- In moderately populated livestock areas, the Vac_live and EU strategies are preferred over the other control strategies.

- In densely populated livestock areas, the Pre strategy is preferred over Vac_live strategy and Vac_kill.

Difference in ranking between clusters of countries, comprising regions with comparable density and/or trade characteristics, are possibly underexposed due to the use of 'average' CVO judgements. Disaggregating the panel of CVOs into subgroups according to the density and trade characteristics of the country represented by the CVOs, followed by an analysis per cluster would provide better insight into the possible presence of alternative rankings.

Individual CVOs or - in general - individual interest groups often differ in their views of the relative importance of the various criteria. Using the MCA framework 
to examine how ranking of alternatives might change under different preferences or weighting systems can show that, for instance, two alternatives always come out best. Their preference order, however, may differ. If the differences between these best alternatives under different weighting systems are rather small, accepting a second best option can be shown to be associated with little loss of overall benefit, as demonstrated by the following example.

The results of the questionnaire demonstrate variation in preferences among three studied interest groups or stakeholders (viz., CVO group, agricultural interest group and non-agricultural interest group). Table 6 summarizes the indicated preference weights for the main criteria per interest group. This overview stresses the contrast in perspectives of the non-agricultural interest group vis-à-vis the other interest groups.

Table 6. Criterion preference weights (\%) per interest group

\begin{tabular}{lccc}
\hline Interest group & \multicolumn{3}{c}{ Criterion } \\
\hline & Epidemiology & Economics & Social ethics \\
CVO & 53 & 30 & 17 \\
Agricultural & 49 & 33 & 18 \\
Non-agricultural & 51 & 15 & 35 \\
\hline
\end{tabular}

An evaluation of the overall dominance scores based on the preference weights of these individual interest groups enables for an examination of the differences in ranking of alternatives. Table 7 shows for each of the interest groups, the overall scores of AI control alternatives for an exporting, densely populated EU member state. Based on the preferences of the CVO and the agricultural interest groups the Pre strategy is ranked first, followed by the Vac-live strategy as second best alternative. From the non-agricultural point of view, the ranking of these two alternatives is just the opposite. However, differences between first and second best alternatives are rather small. The loss of overall benefit associated with the acceptance of the second best alternative is highest for the non-agricultural interest group (difference of 5.8).

Table 7. Overall dominance scores of AI control alternatives based on the criterion weights of the individual interest groups. Bold printed values reflect alternatives with highest scores (= highest rank)

\begin{tabular}{lcccc}
\hline Interest group & \multicolumn{3}{c}{ Control alternative } & $\begin{array}{c}\text { Difference with } \\
\text { second best } \\
\text { alternative }\end{array}$ \\
\hline & Pre & Vac_live & Vac_kill & \\
CVO & $\mathbf{8 . 3}$ & 7.4 & -15.6 & 0.9 \\
Agricultural & $\mathbf{8 . 2}$ & 6.8 & -15.0 & 1.4 \\
Non-agricultural & 4.2 & $\mathbf{1 0 . 0}$ & -14.2 & 5.8 \\
Veterinarian & 7.4 & $\mathbf{8 . 0}$ & -15.4 & 0.6 \\
\hline
\end{tabular}


Generally, when opposing stakeholders discuss alternative options, they quickly focus on their differences of opinions, ignoring the effect of many criteria on which there is an agreement. The MCA technique provides a more balanced approach to ensure that all criteria enter the evaluation, with the result that overall differences are not as great as they seem in an unstructured, face-to-face meeting.

\section{Application of MCA in the field of plant disease control}

Comparable to the control of animal diseases, decision making in quarantine plant diseases is also complex and conflicting, due to the involvement of various epidemiological, economic and social-ethical value judgments. Control measures as the use of a particular pesticide may limit the spread of the infectious disease (= epidemiological value), but could also affect the subsistence of harmless organisms (= social-ethical value), result in residues in potential food products (= economic and social-ethical value) or even influence the existence of a whole ecosystem (= social-ethical value). Controlling the disease by a measure as complete destruction of plants and plant products may be very efficient from an epidemiological point of view, but could have serious economic consequences for the affected producers and - depending on the magnitude of the outbreak - even affect the world food supply, resulting in a global social-ethical distress.

Based on the findings within the described study it can be concluded that the MCA technique could be a suitable tool to assist plant control decision making by providing structure to debates, ensuring quality conversations, documenting the process of analysing the decision, separating matters of fact from matters of judgment, making value judgments explicit, bringing judgments about trade-offs between conflicting objectives to the attention of decision makers, creating shared understanding about the issues, generating a sense of common purpose, and gaining agreement.

\section{REFERENCES}

Charnes, A., Cooper, W.W. and Ferguson, R.O., 1955. Optimal estimation of executive compensation by lLinear programming. Management Science, 1 (2), 138-151.

Churchill, G.A., 1995. Marketing research: methodological foundations. 6th edn. Dryden Press, Fort Worth.

Dodgson, J., Spackman, M., Pearman, A.D., et al., 2000. Multi-criteria analysis manual. Department of the Environment, Transport and Regions, London. [http://www.communities.gov.uk/pub/252/ MulticriteriaanalysismanualPDF1380Kb_id1142252.pdf]

Huirne, R., Van Asseldonk, M., De Jong, M., et al., 2005. Prevention and control of Foot and Mouth Disease, Classical Swine Fever and Avian Influenza in the European Union: an integrated analysis of epidemiological, economic and social-ethical aspects. EU-Research Report. [http://www.warmwell.com/04dec18brusselsconf.html]

Huirne, R.B.M. (ed.) 2002. MKZ: verleden, heden en toekomst: over de preventie en bestrijding van MKZ. LEI, Den Haag. Rapport / LEI. Domein 6, Beleid no. 6.02.14 [http://www2.lei.wur.nl/publicaties/PDF/2002/6_xxx/6_02_14.pdf]

Nijkamp, P., Rietveld, P. and Voogd, H., 1990. Multicriteria evaluation in physical planning. NorthHolland, Amsterdam. Contributions to Economic Analysis no. 185.

Rehman, T. and Romero, C., 1993. The application of the MCDM paradigm to the management of agricultural systems: some basic considerations. Agricultural Systems, 41 (3), 239-255. 
Romero, C. and Rehman, T., 2003. Multiple criteria analysis for agricultural decisions. 2nd edn. Elsevier, Amsterdam. Developments in Agricultural Economics no. 11.

Voogd, J.H., 1982. Multicriteria evaluation for urban and regional planning. PhD Thesis. TU Eindhoven. [http://alexandria.tue.nl/extra1/PRF4A/8203510.pdf] 